\title{
Multicentric Invasive Pleomorphic Lobular Carcinoma of Breast - An Uncommon Histopathological Variant
}

\author{
Sunil V Jagtap*, Garima Agarwal and Swati S. Jagtap \\ Department of Pathology, KIMS University, Karad-415110, Maharashtra, India.
}

\section{ABSTRACT}

Invasive Pleomorphic Lobular Carcinoma is a very rare histopathological variant of breast cancer. It constitutes less than $1 \%$ of invasive breast carcinoma. We present a 70 year female presented with lump in right breast of 10 months duration. The lump was insidious in onset, progressive in nature and was associated with gradual skin color changes. Patient was a known diabetic and was on regular treatment. On fine needle aspiration cytology showed positive for carcinoma cells. Right modified mastectomy was performed. On histopathology reported as Invasive Pleomorphic Lobular Carcinoma - Grade II with multicentric nodules. Right axillary lymph nodes with perinodal infiltration of tumor were noted.

We are presenting this case for its uncommon type, clinical, histomorphological and immunohistochemistry pattern.

Keywords: Breast cancer, Lobular carcinoma breast, Alveolar pattern.

\section{Introduction}

Invasive Pleomorphic Lobular Carcinoma (IPLC) is one of the distinctive subtypes of invasive breast carcinoma. IPLC was first described by Martinez V and Azzopardi in 1979, ${ }^{[1]}$ and later on in 1987 by Page and Anderson. ${ }^{[2]}$ Invasive pleomorphic lobular carcinoma represents less than $1 \%$ of invasive carcinomas. ${ }^{[3]}$ Unlike the classic variant, the tumor cells of the pleomorphic variant of ILC are larger in size , large irregular, pleomorphic, hyperchromatic nuclei with occasional prominent nucleoli and having abundant eosinophilic cytoplasm. It is considered to be an aggressive tumor with poor prognosis.

\section{Case Report}

A 70 year female presented with lump in right breast of 10 months duration. The lump was insidious in onset, progressive in nature and was associated with gradual skin colour changes. Patient was a known diabetic and was on regular treatment. On fine needle aspiration cytology, showed positive for carcinoma cells. Systemic examination was within normal limits. On X-ray and ultrasonography abdomen \& pelvis, no evidence of metastasis was noted. On gross examination a specimen(figure-1) of right modified mastectomy measuring $24.1 \times 22 \times 2.0 \mathrm{~cm}$ and weighing $817 \mathrm{gm}$. Covering skin flap measures $16.5 \mathrm{x}$ $14.5 \mathrm{~cm}$. Tumor was reaching upto the nipple and areola on gross examination. Serial cut sections through the specimen, revealed a grey white, firm tumor measuring 10 x $4.5 \times 2 \mathrm{~cm}$, located in subareolar region. Tumor showed infiltrating borders. Tumor was reaching upto the deep surgical margin. Overlying skin showed discoloration (areas of hyperpigmentation and hypopigmentation) and appeared fixed to the underlying tumor grossly. Nearest peripheral surgical margin was $3.5 \mathrm{~cm}$ away from tumor.

Adjacent breast tissue showed four nodular masses. Largest measuring $1.5 \times 1 \times 0.3 \mathrm{~cm}$ and smallest measuring $0.4 \mathrm{~cm}$ in maximum diameter. Cut section of which revealed grey white firm tumor .Serial sectioning showed three lymph nodes in axillary region ( along the specimen ), largest measuring $1.2 \times 0.8 \times 0.3 \mathrm{~cm}$. Cut section of which was grey white and firm. Serial sectioning through right axillary dissection revealed 06 lymph nodes. Largest lymph node measuring $0.5 \times 0.4 \times 0.1 \mathrm{~cm}$. Cut section of which appears grey white, firm.

On microscopic examination showed a tumor is composed of neoplatic cells arranged in cords, Indian file pattern, small nests, and alveolar pattern and scattered diffusely and multifocally in the breast parenchyma. Individual cells were round having moderately pleomorphic hyperchromatic or vesicular nuclei with 0-2 nucleoli and moderate to ample amount of eosinophilic cytoplasm. In areas targetoid appearance was noted around the terminal duct. Mitotic activity was increased. Tumor was seen diffusely infiltrating in single file with dense fibrovascular stroma separating the tumor.(Figure-2 A,B,C,D.)

On histopathological examination, it was reported as Invasive Pleomorphic Lobular Carcinoma Areas of classical and alveolar pattern were noted. Tumor with multicentric nodules and extensive axillary lymph node with perinodal infiltration of tumor was evident. 
The immunohistochemistry study showed positivity for ER, PR and negativity for HER-2neu.

Our patient received treatment of right sided modified radical mastectomy with axillary clearance. She was kept on chemotherapy. On follow up patient responded well to treatment.

\section{Discussion}

Infiltrating lobular carcinoma (ILC) of the classic type is well recognized type of invasive breast carcinoma. The less well appreciated is a group of variant forms of ILC includes solid, alveolar, mixed, apocrine, pleomorphic , signet-ring, histiocytoid, and tubulo-lobular variants. ${ }^{[1,4]}$

IPLC of breast is a very rare malignancy, which account for less than $1 \%$ of invasive carcinomas. The histomorphological features for diagnosis are pattern of classic lobular carcinoma i.e. invasive tumor with frequently single linear row (Indian file) in dense stroma, targetoid appearance around terminal ducts. Tumor cells are usually round with mild nuclear pleomorphism. IPLC

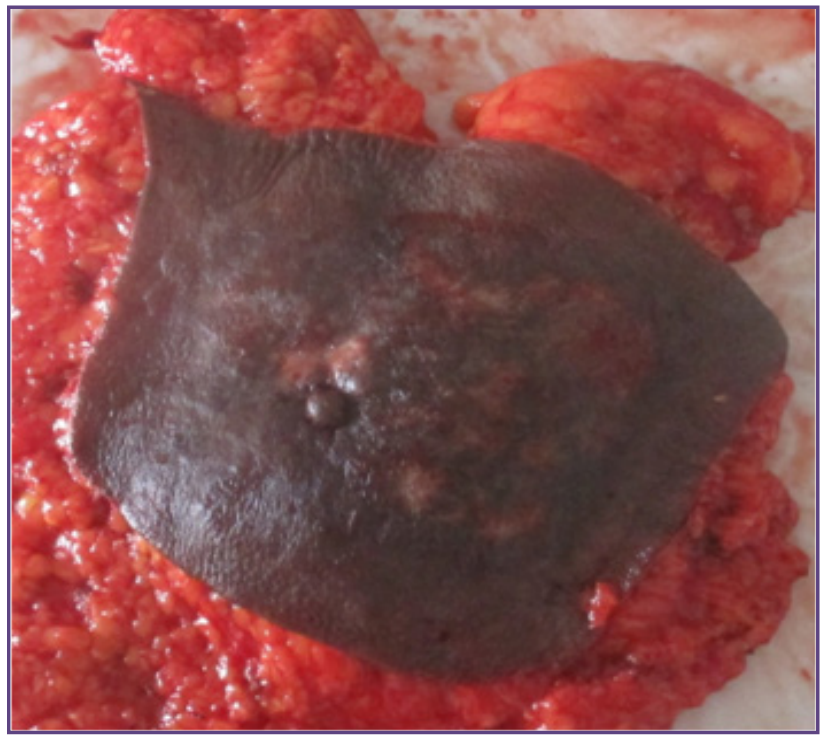

Fig. 1: Gross specimen of right modified mastectomy- the overlying skin showed discoloration, nipple retraction and fixed to the underlying tumor.

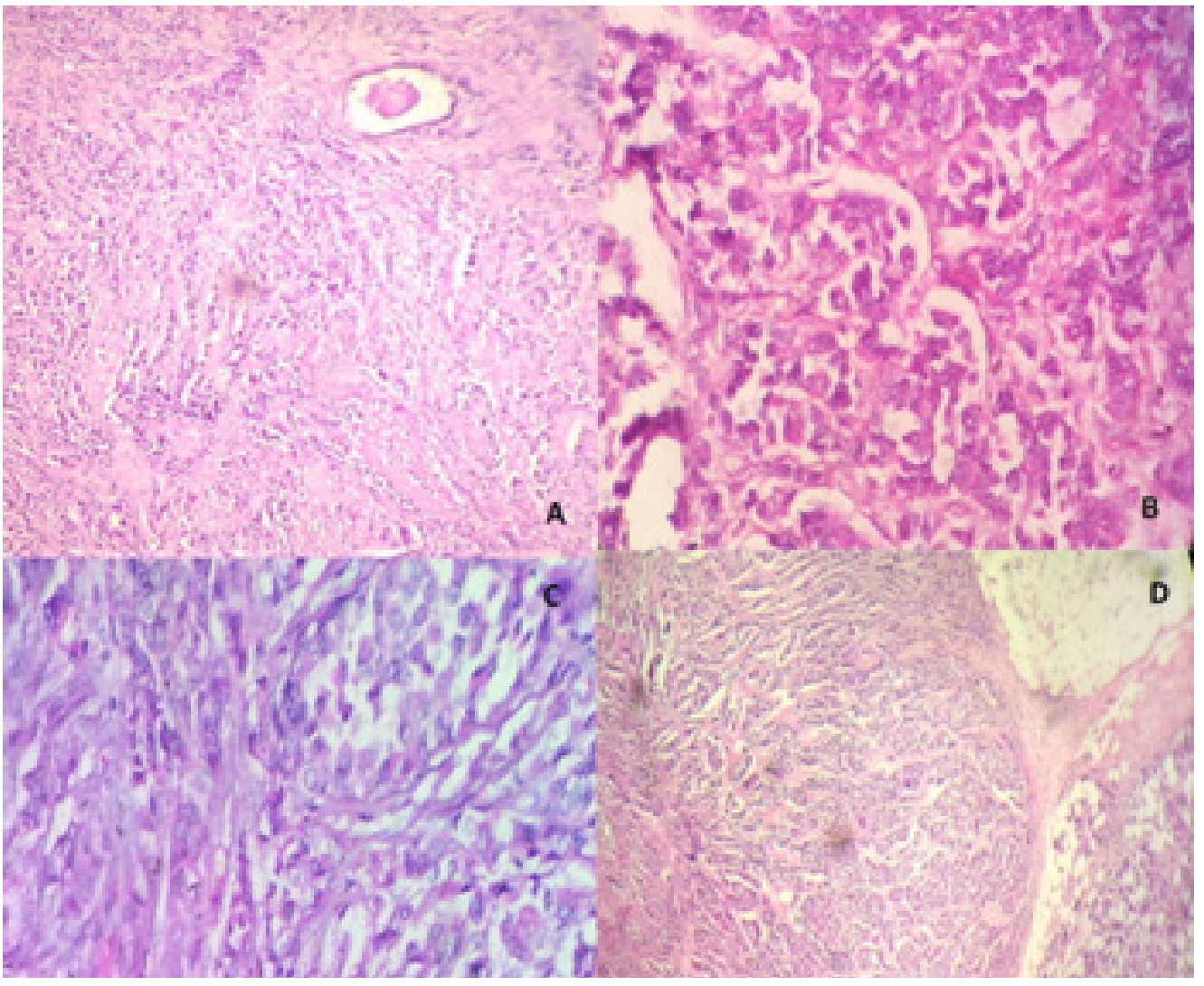

Fig. 2A: Photomicrograph showing Lobular carcinoma showing morphologic pattern of classic and pleomorphic type. Hematoxylin and Eosin, 100 × Figure 2B.- Photomicrograph showing invasive pleomorphic lobular carcinoma ,Hematoxylin\& Eosin, 100x Figure 2C: Photomicrograph showing invasive pleomorphic lobular carcinoma solid, alveolar pattern ,Hematoxylin \& Eosin, 200× Figure 2D : Photomicrograph showing nodule with invasive pleomorphic lobular carcinoma ,Hematoxylin \& Eosin, 100x. 
shows tumor cells are of high nuclear grade II or III and having solid, alveolar pattern.

IPLC on clinical presentation are usually large in size as compared with Invasive breast carcinoma (NOS). The mean size of tumor for IPLC was $3.2 \mathrm{~cm}$ while for IBC (NOS) is $2.2 \mathrm{~cm}$. ${ }^{[5,6]}$ The postmenopausal women are usually affected. In our case tumor was very large $(10 \mathrm{x}$ $4.5 \times 2 \mathrm{~cm}$ ) with multicentric nature. On mammography showed tumor mass with speculated lesion. Mammograms of pleomorphic breast cancer tumors will tend to show well-circumscribed or speculated mass lesions without evidence of calcification.

The FNAC finding in our case showed positive for carcinoma cells. However, differentiation from IBC and ILC is not possible. The study by Jagtap et al showed ILC with $84.6 \%$ ER ,PR positivity and HER-2 neu $23 \%$ positivity on immunohistochemical study ${ }^{[7]} \mathrm{On}$ IHC study our patient was ER, PR positive while HER-2Neu negative. PLCs were frequently negative for ER, PR and E-cadherin; and positive for $\mathrm{p} 53$ and HER-2 in few cases. ${ }^{[8]}$

PLBC is highly aggressive and usually presents as a grade II to III tumor ${ }^{[9]}$ At time of presentation may show evidence of distant metastasis. IPLC is associated with worse prognostic factors when the tumor size is larger and axillary metastasis compared with other types of lobular carcinomas. ${ }^{[9]}$ There is a higher risk of recurrence and decreased survival rate.

\section{Conclusion}

We are presenting this case of invasive pleomorphic lobular carcinoma for its uncommon type, clinical, histomorphological and immunohistochemical pattern. Our case showed multifocal tumor with extensive lymph nodal metastasis and aggressive clinical behavior.

\section{References}

1. Martinez V, Azzopardi JG. Invasive lobular carcinoma of the breast: incidence and variants. Histopathology. 1979;3:467488.

2. Page DL, Anderson TJ. Diagnostic Histopathology of the Breast. Edinburgh: Churchill Livingstone; 1997.

3. Riva C, Dainese E, Caprara G, et al. Immunohistochemical study of androgen receptors in breast carcinoma. Evidence of their frequent expression in lobular carcinoma. Virchows Arch. 2005;447(4):695-700.

4. Dixon JM, Anderson TJ, Page DL, Lee D, Duffy SW. Infiltrating lobular carcinoma of the breast. Histopathology. 1982;6:149-161.

5. Jacobs M, Fan F, Tawfik O. Clinicopathologic and biomarker analysis of invasive pleomorphic lobular carcinoma as compared with invasive classic lobular carcinoma: an experience in our institution and review of the literature. Ann Diagn Pathol. 2012;16:185-189.

6. Orvieto E, Maiorano E, Bottiglieri L, Maisonneuve P, Rotmensz N, Galimberti V, et al. Clinicopathologic characteristics of invasive lobular carcinoma of the breast: results of an analysis of 530 cases from a single institution. Cancer. 2008;113:1511-1520.

7. Sunil V Jagtap, Atul Beniwal, PG Chougule, Heena P Shah, Swati S Jagtap. Invasive Lobular Carcinoma of Breast Histopathological Subtypes: Clinicopathological Study. International Journal of Health Sciences and Research (IJHSR). 2016; 6(7): 105-111.

8. Simpson PT, Reis-Filho JS, Lambros MBK, et al. Molecular profiling pleomorphic lobular carcinomas of the breast: evidence for a common molecular genetic pathway with classic lobular carcinomas. J Pathol. 2008;215:231-244.

9. Sahin S, Karatas F, Erdem GU, Hacioglu B, Altundag K. Invasive pleomorphic lobular histology is an adverse prognostic factor on survival in patients with breast cancer. Am Surg. 2017;83(4):359-364.

*Corresponding author:

Dr. Sunil V Jagtap, Professor, Department of Pathology, KIMS University, Karad-415110, Maharashtra, India

Phone: +91 : 9960628672

Email: drsvjagtap@gmail.com

Financial or other Competing Interests: None. 